\title{
PSICOLOGIA AMBIENTAL: UM ESBOÇO SOBRE O SEU DESENVOLVIMENTO EM PORTUGAL E NO MUNDO
}

\author{
Maria Luísa Lima \\ Elena Sautkina
}

\section{O Espaço na Psicologia e a falta que ele faz}

Um dos resultados mais interessantes da Psicologia consiste na constatação da nossa contínua subestima do impacto do ambiente sobre a nossa maneira de pensar, sentir e de agir. Ignorar a importância das forças da situação na explicação dos comportamentos (Ross, 1977; Jones \& Harris, 1967) e sobrestimar o grau de controlo que temos sobre a nossa vida (Langer, 1982; Taylor \& Brown, 1988) são duas faces de uma mesma moeda que, salientando a liberdade pessoal e as diferenças individuais, é fundamental na visão do senso comum sobre a Psicologia. E, no entanto, dois ramos da Psicologia foram construídos exactamente na rejeição dessa posição e salientando o impacto no comportamento humano da interacção com os outros (no caso da Psicologia Social) e do contexto físico (no da Psicologia Ambiental). Não é estranho, por isso, que, desde a sua origem, constatemos uma grande cumplicidade entre estas duas áreas da Psicologia. Também este número especial da Psicologia é construído na sua maioria com textos de psicólogos ambientais e sociais e reúne contributos que ilustram diferentes dimensões da relação do homem com o seu ambiente, centrado no tema de espaço, risco e comportamento social. Sendo o primeiro número temático da Psicologia a dedicar-se às questões ambientais, considerámos pertinente salientar, de maneira esquemática, a evolução das ideias sobre o ambiente e dos estudos psicoambientais no mundo e em Portugal para depois nos centrarmos no tema deste número e apresentarmos os artigos que inclui.

O adjectivo "ambiental" associado ao substantivo "Psicologia" tanto inclui a Natureza e a sua preservação, como os espaços construídos pelo homem. Curiosamente, apesar de as questões ambientais terem tido início numa tentativa de preservação da natureza, como veremos, a Psicologia Ambiental começou por se dedicar ao impacto do ambiente construído. A 
Psicologia Ambiental inclui também tanto o estudo dos impactos do espaço físico sobre o comportamento humano, como o estudo das próprias ideias socialmente partilhadas sobre o ambiente físico (desde as ideias ambientalistas até à percepção de riscos ambientais). Como veremos, em Portugal, esta última abordagem tem conhecido um desenvolvimento mais acentuado.

\section{O desenvolvimento da Psicologia Ambiental no mundo ${ }^{1}$}

Os movimentos ambientalistas são um fenómeno do século XX. Começaram associados aos grupos conservacionistas, que, no século passado, promoveram a criação das primeiras áreas naturais protegidas nos Estados Unidos e, mais tarde, na Europa. Estes movimentos eram integrados por membros de elites intelectuais, que se preocupavam com o crescimento da civilização e queriam preservar intacta a natureza selvagem (Grove, 1992; Giddens, 1995). Só nos anos de 1970 assistimos à sua constituição como verdadeiros movimentos, com preocupações mais alargadas do que a conservação da natureza (Clapp, 1994). A divulgação deste movimento junto do grande público não foi imediata (ver também Castro, 2000; Melo e Pimenta, 1993; Schmidt, 1999). Schoenfeld, Meier e Griffin (1979) consideram-no como um processo social no qual é possível reconhecer diversas fases: o primeiro impulso foi dado pela academia e pelos cientistas, depois por grupos de interesses e organizações governamentais e, mais tarde, pelos meios de comunicação social que o divulgam junto do grande público.

Desastres ecológicos nos Estados Unidos, tendo por origem as acções do homem, contribuíram fortemente para o nascimento de um interesse pelo ambiente. De facto, nos anos de 1950 e de 1960, diversas áreas científicas vêm, de forma organizada, demonstrar a dependência do homem relativamente ao meio ambiente. Os conceitos de ecossistema e de ecologia, surgidos no século XIX, tornam-se proeminentes nas ciências naturais (Bowler, 1992).

A abordagem científica da dependência entre o homem e o ambiente chega também às ciências sociais. É assim que o conceito de ecologia social se torna heurístico em Sociologia (McKenzie, 1924; Wirth, 1945) e em Psicologia (Lewin, 1936, 1951; Barker, 1965) e que começam a aparecer especializações destas ciências no domínio do ambiente (como a Psicologia Ecológica). Mas a pesquisa neste domínio da Psicologia desenvolve-se claramente a partir dos anos 50. Com efeito, depois da Segunda Guerra Mundial, as ciências sociais (e nomeadamente a Psicologia Social) foram leva-

\footnotetext{
${ }^{1}$ Para uma análise mais detalhada desta evolução e da sua articulação com o ambientalismo, ver Lima, 2002.
} 
das a resolver múltiplos problemas emergentes, dos quais se destaca a relação que as pessoas mantinham no quotidiano com o ambiente construído (Proshansky, 1987). A densidade espacial, edifícios e habitações inadaptados aos utilizadores, o ambiente degradado e destruído eram boas razões para estudar a qualidade de vida nas cidades. A parceria com arquitectos, que começou na construção de edifícios mais adequados às necessidades dos utilizadores, deu origem à conferência "Architectural Psychology and Psychiatry", em 1961, e, mais tarde, ao que se começou por chamar Architectural Psychology. Naquela época ainda não existiam em Psicologia teorias explicativas, conceitos ou pesquisas aplicados à relação indivíduo-ambiente ou às questões ambientais. $\mathrm{E}$, assim, com a iniciativa e o interesse de alguns, surgiu a ideia um pouco utópica de dar início a uma ciência que terá a capacidade de imaginar um ambiente construído novo e feito para o homem (Canter, 2006; Pinheiro, 1997).

Os trabalhos designados claramente como Psicologia Ambiental centraram-se, inicialmente, no impacto das condições ambientais (por exemplo, o ruído) no comportamento humano e só depois se dedicaram à forma como as pessoas avaliam o espaço construído e pensam sobre as relações do homem com a Natureza. Neste sentido, a Psicologia Ambiental ganhou, de maneira inestimável, ao passar da perspectiva determinista ambiental para a perspectiva transaccional. Esta abordagem implica considerar que o impacto do ambiente físico sobre as pessoas é mediado pelos significados socialmente partilhados (Altman \& Rogoff, 1987) e individuais que lhe são atribuídos, salientando, assim, a importância dos processos sociocognitivos nas respostas comportamentais (Nasar, 2000) e o carácter intencional e activo do indivíduo e dos grupos neste processo (Bonnes \& Secchiaroli, 1995). A perspectiva transaccional vem reforçar a face psicossocial da Psicologia Ambiental (ver, por exemplo, Rouquette, Sautkina, Castro, Félonneau \& Guillou-Michel, 2005) e, dará, mais tarde, origem ao estudo das cognições, avaliações e atitudes ambientais e ao aparecimento das teorias de lugar, do conceito da identidade e de ligação ao lugar, aspectos sem os quais, hoje, seria impossível imaginar esta disciplina.

A solidificação da Psicologia Ambiental concretizou-se na organização de um número do Journal of Social Issues, dedicado à Psicologia Ambiental, em 1966, no primeiro artigo sobre Psicologia Ambiental, na Annual Review of Psychology (Craik, 1973), na criação da Environmental Design Research Association (1969), de uma Divisão da APA dedicada à Population \& Environmental Psychology (1973), da International Association for People-Environment Studies (1981), assim como no aparecimento das primeiras revistas especializadas - Environment and Behavior (1969) e Journal of Environmental Psychology (1981). 
A par da incorporação do ambiente nas diversas ciências sociais, das quais a Psicologia Ambiental é um exemplo, no início dos anos 1970 assiste-se à institucionalização das questões ambientais. Os grupos ambientalistas mais conhecidos internacionalmente (Greenpeace e Friends of the Earth) aparecem em 1971. O Dia da Terra é celebrado pela primeira vez em 1970, ano em que foi criada a EPA (Environmental Protection Agency), nos Estados Unidos. O ano de 1972 aparece como um marco na formalização das preocupações ambientais. Ao nível da Comunidade Europeia, o Encontro de Paris marca a primeira discussão do ambiente como um objecto ao nível comunitário, sendo publicado, em 1973, o $1 .{ }^{\circ}$ Programa de Acção sobre o Ambiente. Foi ainda em 1972 que foi publicado o livro Os limites do crescimento (Meadows et al., 1972) e que se realizou, em Estocolmo, a primeira Conferência da ONU sobre o Ambiente, que contou com a participação de 114 países (entre os quais Portugal) e se discutiram não só as questões ambientais, mas também as associadas ao desenvolvimento ${ }^{2}$. É igualmente a partir desta altura que começam a ser criados, em vários paises, órgãos governamentais nacionais especializados no ambiente (como Secretarias de Estado ou Ministérios do Ambiente).

A mediatização das questões ambientais foi um passo fundamental para a divulgação das ideais ambientalistas junto do público. A transmissão quase em directo da fuga de gás radioactivo ocorrida na central nuclear americana de Three Miles Island, em Março de 1979 (Houts, Cleary \& Hu, 1988), por exemplo, contribuiu para uma mobilização do público muito superior do que outros acidentes nucleares de maiores dimensões, ocorridos antes. O tipo de cobertura dos meios de informação foi-se modificando, pássando para a abordagem de problemas de natureza mais global (Hanningan, 1995). Esta atitude dos media, além de tornar possível a sensibilização do público para as questões ambientais, faz progressivamente dos meios de comunicação uma fonte de educação e de informação ambiental e de mudança nas atitudes e comportamentos dos cidadãos (Dunlap \& Van Liere, 1978).

Hoje, tal como as questões ambientais estão socialmente estabelecidas como relevantes, a Psicologia Ambiental apresenta-se como um campo de estudo científico de pleno direito, orgulhando-se dos seus cruzamentos com a Arquitectura, Urbanismo, Geografia Humana, Ecologia e Sociologia. Vejamos agora como se processou esta evolução, em Portugal.

\footnotetext{
2 Desta importante conferência internacional nasceu o Programa das Nacões Unidas para o Meio Ambiente (UNEP: United Nations Environment Programme).
} 


\section{O desenvolvimento da Psicologia Ambiental em Portugal}

Em Portugal, os percursos do ambientalismo e da Psicologia Ambiental podem ser descritos nas mesmas fases em que dividimos a sua evolução internacional. Mansinho e Schmidt (1994) situam a sua génese nos estudos rurais, onde investigadores como Orlando Ribeiro, Amorim Girão ou Leite de Vasconcellos foram responsáveis por um conjunto de trabalhos inovadores em Portugal, em que o comportamento social é interpretado em função dos atributos morfológicos regionais. Os movimentos conservacionistas têm uma expressão na criação da Liga para a Protecção da Natureza, em 1948, a associação de defesa do ambiente mais antiga da Península Ibérica. Trata-se de um movimento com as mesmas características dos seus congéneres noutras partes do mundo: muito ligado à academia e a elites intelectuais, que foram, aliás, responsáveis pela elaboração dos estudos técnicos que levaram à criação das primeiras áreas protegidas em Portugal (Melo e Pimenta, 1993). Mas foi principalmente durante o período Marcelista do Estado Novo que assistimos, a par de um claro empenhamento na industrialização do país, ao emergir de uma preocupação com as questões ambientais. Neste contexto, em 1971 é criada a Comissão Nacional do Ambiente (CNA) para preparar a participação portuguesa na Conferência de Estocolmo. Todavia, só após a implantação do regime democrático se dá a criação de órgãos executivos claramente responsáveis pelas questões ambientais (em 1974, é criada a Secretaria de Estado do Ambiente) e só no fim dos anos de 1980 se começa a delinear uma estratégia mais coerente neste domínio (Schmidt, 1999). Em paralelo a este esforço de institucionalização dos órgãos responsáveis pelas questões ambientais, assiste-se, nos anos de 1980, à criação das associações ambientalistas (o Geota, em 1981, e a Quercus, em 1985).

Tal como sucedeu noutros países, os meios de comunicação social começam, após este período de institucionalização das questões ambientais, a dar-lhes ênfase noticioso. O maior interesse dos media pelos problemas ambientais nacionais é contemporâneo do aparecimento de canais privados de televisão e da reorganização do sector da imprensa, onde alguns jornais dão voz a mobilizações ambientais, a denúncias ou queixas dos cidadãos (Schmidt, 1999). Em início dos anos de 1990, começam igualmente a surgir as primeiras revistas especializadas no domínio do ambiente: Indústria e Ambiente, em 1993, e Forum Ambiente, em 1994.

Se o compararmos com o panorama internacional descrito acima, o movimento ambientalista em Portugal revela um atraso de algumas décadas. Gostaríamos apenas de salientar que este atraso é ainda mais acentuado ao nível das ciências sociais. Apesar de datar dos anos 1970 a formalização de especializações ambientais de diversas ciências sociais, isso só se verificou em Portugal, a partir dos finais dos anos de 1980 e dos anos 1990. Tal de- 
veu-se à inexistência de formação em ciências sociais antes da instauração de um Estado democrático em Portugal.

De qualquer modo, é de salientar a criação do Grupo de Ecologia Social, em 1984, fruto da investigação pioneira no domínio da Psicologia Ecológica e Ambiental de Luís Soczka (1983a, 1984, 1989; Soczka et al., 1985). No lugar de excelência da produção da investigação das ciências da engenharia sobre a construção (o Laboratório Nacional de Engenharia Civil), este psicólogo conseguiu fazer vingar um espaço de investigação em ciências sociais de tradição inter-disciplinar sobre os efeitos do ambiente no comportamento social. No seu trabalho pioneiro encontramos pesquisa nas diversas áreas relevantes da Psicologia Ambiental. A sua investigação sobre o ruído nas imediações do aeroporto de Faro mostra, numa perspectiva claramente transaccional, como a incomodidade acústica está dependente do significado atribuído à fonte de ruído (Soczka, 1983a). Contribuiu também para a solidificação, em Portugal, da área da avaliação do ambiente, desenvolvendo uma escala de atitudes sociais e políticas (a SOPOL) na qual incluiu uma escala de atitudes ambientais (Soczka, 1983b).

Nos diversos trabalhos em que reflectiu sobre o ambiente urbano (Soczka, 1984, 1988, 2005), teve sempre a preocupação de aliar uma perspectiva ecológica e pluridisciplinar à procura de aplicações dos seus resultados. A sua linha de pesquisa sobre o bairro da Musgueira Sul (Soczka et al., 1985; Soczka, 1989), utilizando a abordagem da Ecologia Social na compreensão das relações no bairro e no desenvolvimento infantil (Soczka et al., 1988), marcou uma geração de investigadores que por ali passou, teve impacto nas políticas de realojamento da Câmara de Lisboa e promoveu a interdisciplinaridade. O seu impacto na investigação neste domínio foi visível em Portugal com a organização do Colóquio Viver (n)a Cidade, realizado no LNEC, em 1990, numa colaboração do Grupo de Ecologia Social com o Núcleo de Estudos Urbanos e Territoriais do ISCTE. Mas também foi relevante internacionalmente, ao organizar no LNEC, juntamente com David Canter, Correia Jesuíno e Geophrey Stephenson, um encontro sobre Environmental Social Psychology, que reuniu em Lisboa, em 1986, alguns dos investigadores mais relevantes neste domínio (Canter, Jesuíno, Soczka \& Stephenson, 1988).

Actualmente, continuando o campo aberto por Luís Soczka, diversas pessoas trabalham em Portugal no domínio da Psicologia Ambiental. Para além das que fazem parte deste número, lembramos os trabalhos de José Manuel Palma nas questões de impacto e stress ambiental (Palma-Oliveira, 1992) e de Constança Paúl na avaliação da qualidade ambiental de ambientes institucionais (Paúl, 2005). 
Moser e Uzzell afirmam que a Psicologia Ambiental contemporânea estuda "os indivíduos e os grupos no seu contexto físico e social, focando-se em particular no estudo das percepções, atitude, avaliações e represemtações do ambiente, assim como nos comportamentos a eles associados" (2003, p. 422). Em Portugal, temos já pesquisa ilustrativa destes diversos domínios, e este número é uma prova disso.

\section{A apresentação do número temático}

O projecto deste número temático nasceu durante a Escola de Verão em Psicologia Ambiental "Espaços Urbanos e Comportamentos Anómicos", uma organização conjunta do Departamento de Psicologia Social e das Organizações (ISCTE) e do Laboratório de Psicologia Ambiental (Universidade Paris Descartes), que decorreu em Julho 2005 com o apoio da FCT, do programa das acções bilaterais Luso-Francesas, e do Centro de Investigação e de Intervenção Social (ISCTE). O evento reuniu professores e estudantes portugueses e estrangeiros à volta de temas como riscos urbanos (criminalidade, acidentes), medos colectivos e comportamento social. Durante a Escola de Verão, foram organizados workshops de investigação, seminários e conferências que serviam de base a alguns artigos aqui publicados.

Neste número temático, o tema complexo Espaço, risco e comportamento social é abordado de formas bastante diversificadas. Os riscos e as ameaças de que nos falam os autores são variados, incluindo desde os riscos associados ao terrorismo, à criminalidade urbana, aos acidentes, ou à ameaça de transformação do património cultural urbano. Também os contextos ambientais que interessam aos autores deste número são diversos. Assim, encontramos trabalhos com preocupações mais globais, onde está em foco o ambiente urbano em geral; mas também há trabalhos com uma focalização mais específica, em que se examinam bairros ou ambientes institucionais particulares.

Seguimos esta lógica na organização deste número, começando com os artigos mais gerais e terminando nos que se centram em espaços específicos. Assim, no primeiro artigo deste número temático, Michel-Louis Rouquette propõe uma taxinomia dos medos colectivos através de uma análise de dois critérios: "razões" percepcionadas da origem de um objecto de medo e os tipos de locais onde o medo se manifesta. A seguir, Teresa Heitor apresenta uma reflexão teórica sobre um factor de segurança, um potencial inibidor de oportunidades criminosas em ambiente urbano: a vigilância natural. Ainda numa perspectiva mais generalista, o artigo de Luísa Lima, Si- 
bila Marques, Susana Batel, Sandra Carvalho, Juana Haldon e Stéphanie Pornin analisa o optimismo comparativo face a riscos urbanos, tendo em conta um outro factor psicoambiental, a identificação com o lugar, num estudo conduzido em 3 cidades europeias: Lisboa, Huelva e Lyon.

Os restantes artigos deste número referem-se a espaços mais específicos. Rebeca Contreras, Gabriel Moser e Yannick Savina descrevem o impacto que as diferenças socioeconómicas e as do contexto de vida urbana podem ter sobre a relação entre a convivência pública e as percepções de segurança urbana, comparando bairros favorecidos e zonas pobres da cidade de México e da vila mexicana Querétaro. O artigo de Elena Sautkina, Clara António, Hugo Caseira, Filipe Correia, Dimitris Dimitriou, Cláudia Grijó, Ana Loureiro e Rita Morais trata dos factores ambientais e psicossociais da avaliação de risco de furto por carteirista em dois locais particulares em Lisboa (Praça do Comércio e Alfama) que se diferenciam tanto ao nível da especificidade do tecido espacial, como ao nível da composição social e das interacções sociais que aí ocorrem. Paula Castro e Susana Batel levantam questões relativas à participação pública nos projectos de transformação do património histórico urbano, a partir da análise de uma controvérsia sobre a transformação do Convento dos Inglesinhos situado no Bairro Alto em Lisboa, que envolveu os técnicos camarários e os moradores do Bairro. David Uzzell e Jennifer Brown oferecem uma abordagem conceptual de dimensões psicológicas e ambientais do medo de crime (fear of crime) nos utentes das estações ferroviárias, incluindo, particularmente, os seus dois componentes: a probabilidade dos incidentes ameaçadores e o impacto potencial de certos incidentes. Por fim, Elena Sautkina, Michel-Louis Rouquette, Sarah Amador, Christophe Castano e Aimée Casal analisam o comportamento não civilizado enquanto uma consequência de ruptura de ligações sociais entre grupos (empregados, clientes), nos espaços de três empresas francesas: SNCF, La Poste e Galeries Lafayette.

$\mathrm{O}$ número que têm nas mãos não foi feito com o objectivo de envolver toda a diversidade relativa ao tema anunciado, mas, antes de tudo, para ilustrar o interesse de um campo de investigação jovem e produtivo chamado Psicologia Ambiental.

\section{Referências}

Altman, I., \& Rogoff, B. (1987). World-views in psychology: trait, interactional, organismic and transactional perspectives. In D. Stokols \& I. Altman (Eds.), Handbook of environmental psychology (pp. 7-40). New York: Wiley.

Barker, R. (1965). Explorations on ecological psychology. American Psychologist, 20, 1-14. 
Bonnes, M., \& Secchiaroli, G. (1995). Environmental Psychology: A Psycho-Social Introduction. London: Sage.

Bowler, P. J. (1992). The Fontana History of the Environmental Sciences. London: Fontana Press.

Canter, D. (2006). Environics in the wild. Bulletin of People-Environmental Studies, $30,5-10$.

Canter, D., Jesuíno, J. C., Soczka, L., \& Stephenson, G. (Eds.) (1988). Environmental Social Psychology. London: Kluwer Academic Press.

Castro, P. (2000). Natureza, ciência e retórica na construção social da ideia de ambiente: Representações sociais e discursos. Tese de doutoramento não publicada, Instituto Superior de Ciências do Trabalho e da Empresa, Lisboa.

Catton, W., Jr., \& Dunlap, R. E. (1978). Environmental sociology: A new paradigm. The American Sociologist, 13, 41-49.

Clapp, B.W. (1994). The Environmental History Of Britain Since The Industrial Revolution. London: Longman.

Craik, K. (1973). Environmental psychology. Annual Review Psychology, 24, 403$-433$.

Dunlap, R. E., \& Van Liere, K. D. (1978). The New 'Environmental Paradigm': A Proposed Measuring Instrument and Preliminary Results. Journal of Environmental Education, 9, 10-19.

Giddens, A. (1995). As consequências da modernidade. Oeiras: Celta.

Grove, R. H. (1992). Origins of Western Environmentalism. Scientific American (July), 22-27.

Houts, P. S., Cleary, P. D., \& Hu, T.-W. (1988). The Three Mile Island Crisis: Psychological, social and economical impacts on the surrounding population. The Pennsylvania State University Press.

Jones, E. E. \& Harris, V. A. (1967). The attribution of attitudes. Journal of Experimental Social Psychology, 3, 1-24.

Langer, E. J. (1982). The Illusion of Control. In D. Kahneman, P. Slovic, \& A. Tversky (Eds.), Judgment Under Uncertainty: Heuristics and Biases (pp. 231-246). New York: Cambridge University Press.

Lewin, K. (1936). Principles of topological psychology. New York: McGraw Hill.

Lewin, K. (1951). Field theory in social science. New York: Harper \& Row.

Lima, M. L. (2002). O aparecimento das questões ambientais em Portugal - A propósito da apresentação do módulo sobre o Ambiente do ISSP. In L. Lima, M. V. Cabral, J. Vala, \& A. Ramos (Org.), Atitudes Sociais dos Portugueses: Ambiente e Desenvolvimento - Base de dados 4 (pp. 9-24). Lisboa: Imprensa de Ciências Sociais.

Mansinho, M. I., \& Schmidt, L. (1994). A emergência do ambiente nas Ciências Sociais: análise de um inventário bibliográfico. Análise Social, 125/126, 441-481 .

McKenzie, R. D. (1924). The Ecological Approach to the Study of Human Community, American Journal of Sociology, 30 (3), 287-301. 
Meadows, D. H., Meadows, D. L., Randers, J., \& Behrens III, W.W. (1972). The Limits to Growth. New York: Universe Books. [Edição portuguesa de 1973: Os limites do Crescimento, Lisboa: Editorial Pórtico].

Melo, J. J., \& Pimenta, C. (1993). O que é Ecologia?. Lisboa: Difusão Cultural.

Moser, G., \& Uzzell, D. (2003). Environmental Psychology. In T. Million \& M. J. Lerner (Eds.), Comprehensive handbook of psychology (vol. 5, pp. 419-445). New York: John Wiley \& Sons.

Nasar, J. L. (2000). The evaluative image of places. In W. B. Walsh, K. H. Craik, R. H. Price (Eds.), Person-Environment Psychology: New directions and perspectives (2nd ed., pp. 117-168). Mahwah, NJ: Lawrence Erlbaum.

Palma-Oliveira, J. M. (1992). Stress Ambiental: Um selectivo ponto da situação e modelo explicativo. Revista Portuguesa de Psicologia, 28, 13-77.

Paúl, C. (2005). Envelhecimento e Ambiente. In L. Soczka. (Org.), Contextos Humanos e Psicologia Ambiental (pp. 247-268). Lisboa: Fundação Calouste Gulbenkian.

Pinheiro, J. Q. (1997). A Psicologia Ambiental: A busca de um ambiente melhor. Estudos de Psicologia, 2(2), 377-398.

Proshansky, H. M. (1987). The field of environmental psychology: securing its future. In D. Stokols \& I. Altman (Eds.), Handbook of Environmental Psychology (vol. 2, pp. 1467-1488). New York: Wiley.

Ross, L. D. (1977). The intuitive psychologist and his shortcomings: distortions in the attribution process. In L. Berkowitz (Ed.), Advances in Experimental Social Psychology (vol. 10, pp. 173-220). New York: Academic Press.

Rouquette, M. L., Sautkina, E., Castro, P., Felonneau, M. L., \& Guillou-Michel, E. (2005). Social representations theory and environmental studies. In B. Martens, \& A. Keul (Eds.), Designing Social Innovation - Planning, Building, Evaluating (pp. 107-115). Göttingen: Hogrefe \& Huber Publishers.

Schmidt, L. (1999). Ambiente e natureza no ecran: Emissões televisivas, remissões culturais. Tese de doutoramento não publicada, Instituto Superior de Ciências do Trabalho e da Empresa, Lisboa.

Schoenfeld, A. C., Meier, R. F., \& Griffin, R. J. (1979). Constructing a social problem: the press and the environment. Social Problems, 27, 38-61.

Soczka, L. (1983a). Annoyability, noise annoyance and personality. Relatório Técnico M 601. Lisboa: Laboratório Nacional de Engenharia Civil.

Soczka, L. (1983b). SOPOL - Estudo factorial de um questionário de atitudes sociais validado para a população portuguesa. Lisboa: Laboratório Nacional de Engenharia Civil.

Soczka, L. (1984). Espaço urbano e comportamentos agressivos. Da etologia à psicologia ambiental. Relatório Técnico ITI 37. Lisboa: Laboratório Nacional de Engenharia Civil.

Soczka, L. (1988). Ecologia social do risco psicológico em meio urbano. Psicologia, IV (3), 307-346.

Soczka, L. (1989). A perspectiva ecológica em psicologia. Contribuição para o estudo da ecologia social de um bairro da lata. Relatório Técnico NS 54. Lisboa: Laboratório Nacional de Engenharia Civil. 
Soczka, L., Boavida, E., Machado, P., \& Pereira, A. (1985). Ecologia social da Musgueira 1. Análise sociodemográfica e das condições ambientais. Relatório Técnico ITI 52. Lisboa: Laboratório Nacional de Engenharia Civil.

Soczka, L., Boavida, E., Pereira, A., \& Machado, P. (1988). Children of Musgueira: a study on the social ecology of a shanty-town. In D. Canter, J. C. Jesuíno, L. Soczka, \& G. Stephenson (Eds.), Environmental Social Psychology (pp. 106-115). London: Kluwer Academic Press.

Taylor, S. E., \& Brown, J. D. (1988). Illusion and Well-Being - a Social Psychological Perspective On Mental-Health. Psychological Bulletin, 103 (2), 193-210 .

Wirth, L. (1945). Human Ecology. American Journal of Sociology, 50, 483-488. 